\title{
Incubation of Accumbal Neuronal Reactivity to Cocaine Cues During Abstinence Predicts Individual Vulnerability to Relapse
}

\author{
Karine Guillem ${ }^{*, 1,2}$ and Serge H Ahmed A $^{*, 1,2}$ \\ 'Université de Bordeaux, Institut des Maladies Neurodégénératives, UMR 5293, Bordeaux, France; ${ }^{2}$ CNRS, Institut des Maladies \\ Neurodégénératives, UMR 5293, Bordeaux, France
}

\begin{abstract}
An important goal for the treatment of cocaine addiction is to identify neuromarkers that can predict individual vulnerability to relapse after abstinence. There is some evidence that individual reactivity to cue-induced craving may predict subsequent relapse after a period of abstinence. Here we sought to identify the neuronal correlates of this predictive relationship in rats. Rats were trained to self-administer cocaine $(6 \mathrm{~h})$ for 16 days to induce escalation of cocaine intake. Then rats underwent a I-month period of forced abstinence after which they were re-exposed to cocaine self-administration (6h) for 8 additional days to induce re-escalation of cocaine intake. We recorded nucleus accumbens (NAc) neuronal responses to drug conditioned stimuli (CS) I day before and after I month of abstinence from cocaine intake escalation. Rats were ranked according to their individual percentage of CS responsive neurons recorded during the last day of abstinence and split by the median into two groups. We found evidence for a robust, incubation-like increase in NAc reactivity to cocaine cues after abstinence only in a subset of individuals (High CS rats). Importantly, compared with other rats that did not present an incubation of NAc reactivity to cocaine cues (Low CS rats), High CS rats were faster to re-escalate their intake of cocaine after abstinence. In addition, after re-escalation, they worked harder and were less sensitive to risk of punishment than Low CS rats, indicating a strengthened motivation to seek and/or take the drug in that group of rats. Overall, these findings indicate that incubation of NAc neuronal reactivity to cocaine cues during abstinence may constitute a predictive neuromarker for individual vulnerability to relapse.

Neuropsychopharmacology (2018) 43, 1059-1065; doi:I0.1038/npp.2017.224; published online 18 October 2017
\end{abstract}

\section{INTRODUCTION}

An important goal for cocaine addiction research is to identify neuromarkers that can be used as reliable predictors of vulnerability to relapse after prolonged abstinence. There is some evidence that individual reactivity to cue-induced craving may predict subsequent relapse after a period of drug abstinence (Dackis and O'Brien, 2001; Gawin, 1991; Sinha et al, 2000). At the neurobiological level, there is cumulative evidence that individual differences in brain responses to drug-associated cues may predict relapse. However, the origins of these differences in behavioral and neuronal reactivity to drug cues are unclear. In particular, it is not entirely clear whether they originate from initial differences in drug histories and/or some other processes that occur during abstinence. Here we sought to address this question using an animal model of relapse.

Previous research has shown that, like in humans, cues that are closely associated with cocaine self-administration (referred to as conditioned stimuli, CS) can gain through

\footnotetext{
*Correspondence: Dr K Guillem or Dr SH Ahmed, Université de Bordeaux, Institut des Maladies Neurodégénératives, UMR CNRS 5293, | 46 rue Léo Saignât, 33076 Bordeaux, France, Tel:+33 557 57| 566, Fax: +33 556900 278, E-mail: karine.guillem@u-bordeaux.fr or serge.ahmed@u-bordeaux.fr

Received 9 June 2017; revised 25 August 2017; accepted 29 August 2017; accepted article preview online 18 September 2017
}

Pavlovian conditioning the ability to elicit and maintain drug seeking after abstinence (See, 2002; Shalev et al, 2002). More importantly, this conditioned ability can considerably increase with the duration of the abstinence period (Conrad et al, 2008; Grimm et al, 2001; Lu et al, 2004). As no external event or process seems to be required, this timedependent increase in drug-seeking behavior has been attributed to an incubation-like process. At the neurobiological level, this incubation process is associated with an increasing reactivity of accumbal (NAc) neurons to cocaineassociated cues (Conrad et al, 2008; Ferrario et al, 2011; McCutcheon et al, 2011), as well as to an increasing proportion of NAc neurons that phasically respond to cocaine-related behavior (Guillem et al, 2014; Hollander and Carelli, 2005, 2007).

These observations strongly suggest that incubation-like increase in NAc neuronal reactivity to cocaine cues could constitute a predictive neuromarker for subsequent vulnerability to relapse. To address this question, we re-analyzed data from a recently published study (Guillem et al, 2014). Specifically, we looked at individual differences in abstinence-induced incubation of NAc neuronal reactivity to a visual cue that was previously associated with escalation of cocaine intake. Then we looked at how these differences in incubation-like increase in conditioned neuronal reactivity were associated with postabstinence re-escalation of cocaine 
self-administration and associated changes in drug motivation and resistance to risk of punishment.

\section{MATERIALS AND METHODS}

\section{Animal and Surgery}

Male Long-Evans rats $(n=10)$ were surgically prepared with a catheter in the right jugular vein and an array of 16 tefloncoated stainless steel microwires in the NAc core and shell subregions (AP: +0.7 to $+2.7 \mathrm{~mm}$, ML: \pm 0.8 to $\pm 2.2 \mathrm{~mm}$ relative to bregma, and DV: -6.8 to $-7.2 \mathrm{~mm}$ relative to level skull) as previously described in detail elsewhere (Guillem et al, 2014). Rats were initially housed two per cage. After surgery, they were housed individually in a temperature- and ventilation-controlled environment under a reversed $12 \mathrm{~h}$ light/dark cycle.

\section{Experimental Stages and Procedures}

Behavioral procedures were carried out in operant chambers equipped with a retractable lever, a house light mounted on the ceiling, a signal light above the response lever, a white noise-generator, and a tone generator (Med-Associates, St. Albans, VT). Rats underwent three successive stages of drug self-administration: (1) escalation of cocaine intake with extended drug access, (2) forced abstinence, and (3) postabstinence re-escalation of cocaine intake (Figure 1a), as described in detail elsewhere (Guillem et al, 2014). Briefly, during the first stage, rats were allowed to press the operant lever to self-administer cocaine during 16 consecutive 6-h sessions. Session onset was signaled by illumination of the house light and insertion of the retractable lever. Each reinforced lever-press was followed by an intravenous cocaine injection (ie, $0.75 \mathrm{mg} / \mathrm{kg}$ per injection, free base, in $0.2 \mathrm{ml}$ over $7.5 \mathrm{~s}$ ) and was signaled by turning on for $10 \mathrm{~s} \mathrm{a}$ cue light above the lever (conditioned stimuli (CS)). Then rats underwent a 30-day abstinence period during which drug access was interrupted and rats remained in their home cages. Finally, after abstinence, rats were re-exposed to 6-h access to cocaine self-administration, as during the initial escalation stage, for 8 additional days to induce re-escalation of cocaine intake.

\section{Recording Sessions and NAc Neuronal Responses}

In our original study (Guillem et al, 2014), we reported NAc neuronal responses to the cocaine-paired visual cue after escalation of cocaine self-administration but not after abstinence. In addition, these responses were averaged across all individuals. Here we looked more specifically at how NAc neuronal responses to the cocaine cue undergo an incubation-like process after abstinence and how individual variation in this process is associated with subsequent vulnerability to re-escalate cocaine self-administration and associated change in drug motivation and resistance to risk of punishment. To this end, we recorded phasic NAc neuronal reactivity to the cue at different times: (1) during habituation to the self-administration chamber and thus before any drug exposure (Hab); (2) during the last session of cocaine intake escalation (Esc); and, finally, (3) during the
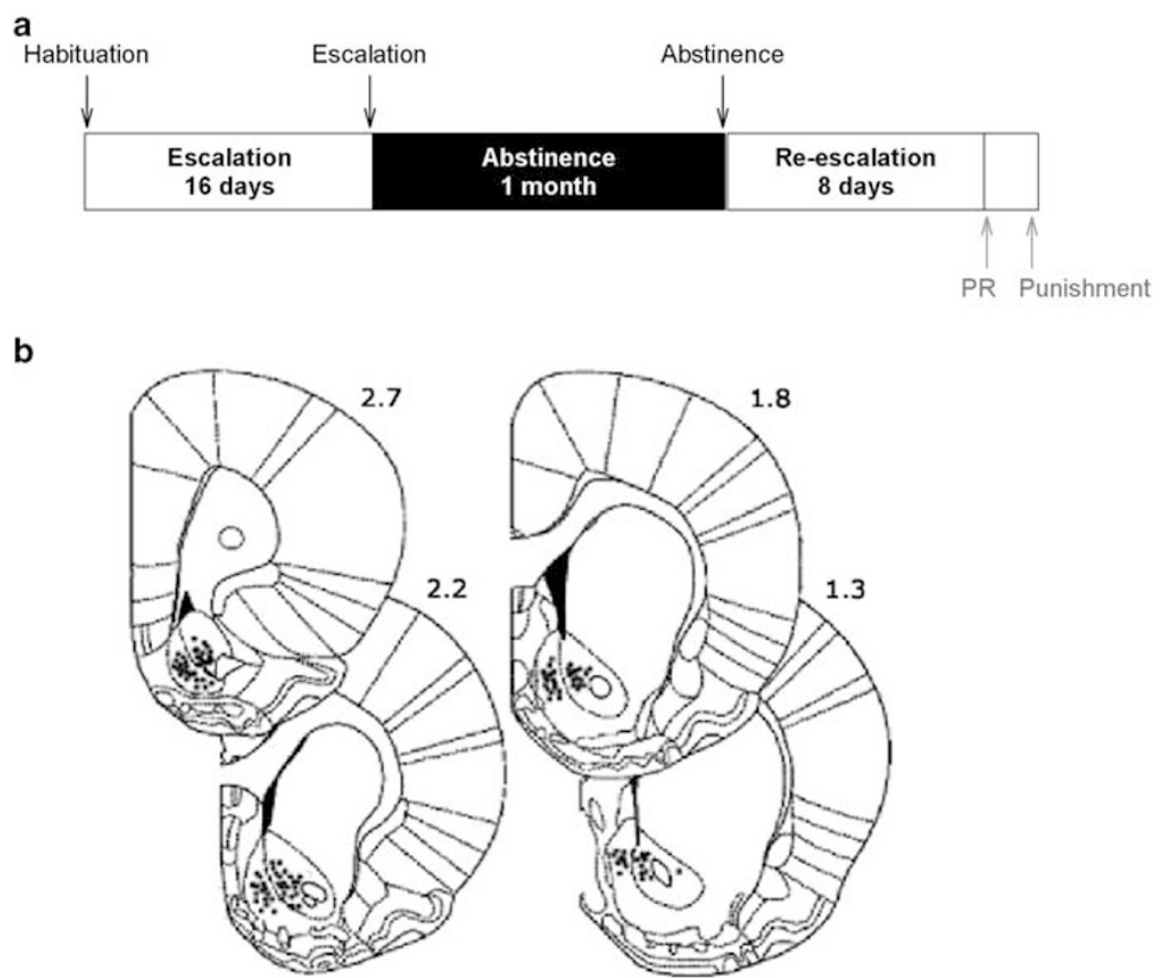

Figure I (a) Schematic of the different phases of the experiment (see Material and Methods section for details). Black arrows indicate the different recording sessions (Habituation, Escalation, and Abstinence). Gray arrows indicate the PR schedule of reinforcement and the punishment session after reescalation postabstinence. (b) Coronal sections showing the location of microwires electrodes from which NAc neurons were recorded. 
last day of abstinence, $24 \mathrm{~h}$ before the re-escalation stage (Abs) (Figure 1a).

Voltage signals from each microwire were recorded, amplified up to $32000 \times$, processed, and digitally captured using commercial hardware and software (Plexon, Dallas, TX) as previously described (Guillem et al, 2014). The average number of electrodes from which NAc neurons were recorded was $7.1 \pm 0.4$. All recording sessions included an initial cue-probe phase to assess NAc phasic neuronal responses to the drug-associated stimuli presented alone (CS responsive neurons). During the cue-probe phase, the CS was presented 10 times on a variable-interval schedule with an average inter-trial interval of $2 \mathrm{~min}$. Each neuron was tested for a phasic change in firing time-locked to the presentation of the CS (CS responsive neurons). Neural phasic responses to the CS were characterized by a significant change in firing during the 10-s CS vs a 10-s control period before CS onset (background) using a $z$-score statistics $(p<0.05)$. $Z$-scores were calculated as $(X-B) / S D$ where $X$ is the mean firing rate during the CS, $B$ is the mean firing rate during the background period, and $\mathrm{SD}$ is the standard deviation of the background activity. For each animal and recording session (ie, Hab, Esc and Abs), we determined the percentage of CS responsive neurons. The percentage of CS responsive neurons was then averaged across rats to obtain a mean $( \pm S E M)$ percentage value. Changes in the percentage of CS responsive neurons were assessed using parametric ANOVA statistical analyses.

Histological procedures were used to identify the location of all wire tips used to record neurons as previously described (Guillem et al, 2014). Coronal sections showing the location of microwires from which NAc neurons were recorded are shown in Figure 1b. Though we tested for an effect of NAc subregion in the present manuscript, the small number of rats per group $(n=5)$ was insufficient for reliable statistical analysis.

\section{Behavioral Measurements of the Motivation For Cocaine}

Progressive ratio procedure. To assess eventual differences in the motivation to self-administer cocaine at the end of the re-escalation stage, all rats were tested under a progressive ratio procedure during one session (PR, Figure 1a). This session was conducted $24 \mathrm{~h}$ after the last session of reescalation. The FR response requirement for reinforcement was increased according to the following quasi-exponential progression: 1, 2, 4, 6, 9, 12, 15, 20, 25, 32, etc. (Richardson and Roberts, 1996). The PR session ended when no drug delivery occurred during $1 \mathrm{~h}$ or after $6 \mathrm{~h}$ has elapsed, whichever came first. The last completed ratio at the end of the session, called the breaking point, was used as an index of cocaine motivation.

Punishment procedure. Resistance to risk of punishment was measured $72 \mathrm{~h}$ after the PR session (Punishment, Figure 1a) (Kearns et al, 2002; Vanderschuren and Everitt, 2004) during 2 consecutive 2-h sessions of cocaine selfadministration: a non-punished session followed $24 \mathrm{~h}$ later by a punished session. The punished session began with a warm-up, non-punishment period during which rats were allowed to self-administer 10 consecutive doses of cocaine under a regular FR1 schedule of reinforcement. Thereafter, completion of the FR requirement resulted in the random delivery of a cocaine injection alone or a cocaine injection plus a mild electric footshock $(0.4 \mathrm{~mA}, 2 \mathrm{~s})$. Thus the risk or probability of punishment was $50 \%$. The effect of punishment was measured by comparing the rate of cocaine selfadministration during the punished session with that during the non-punished session. The difference in drug selfadministration rate between these two sessions served as an operational measure of the persistence of drug taking: the shorter this difference, the higher the persistence.

\section{RESULTS}

NAc neuronal activity was recorded at three different times: (1) during habituation to the self-administration chamber and thus before any drug exposure (Hab); (2) during the last session of cocaine intake escalation (Esc); and, finally, (3) during the last day of abstinence, $24 \mathrm{~h}$ before the reescalation stage (Abs) (Figure 1a). Importantly, in order to determine NAc phasic neural responsiveness to drugassociated stimuli (CS responsive neurons), each recording session was preceded by a cue-probe phase during which the
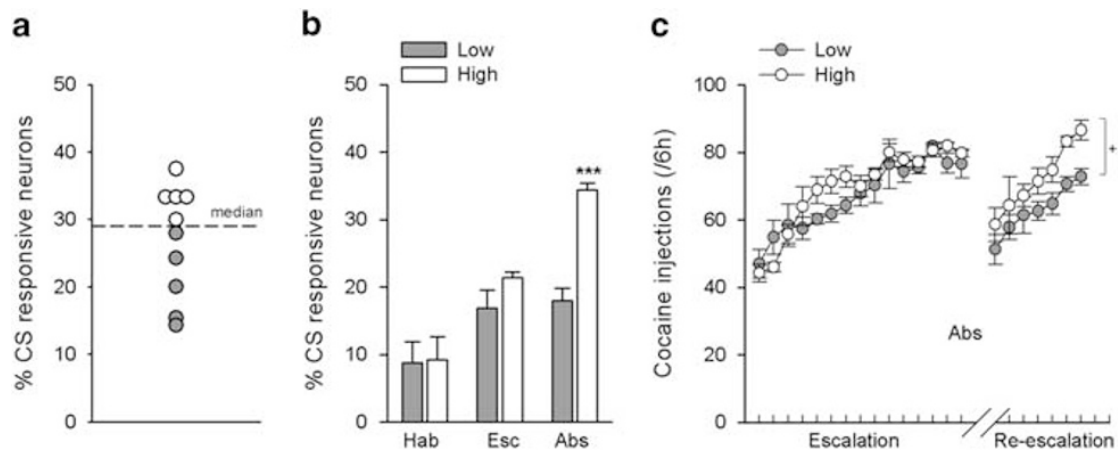

Figure 2 Increased cocaine-taking behaviors in High CS rats. (a) Distribution of individual prevalence of CS responsive neurons after abstinence. Screening analysis of the percentage of CS responsive neurons was conducted by median split into either Low (gray circles) and High CS (white circles) groups ( $n=5 /$ group). (b) Mean ( \pm SEM) percentage of CS responsive neurons in Low (gray bars) and High CS (white bars) rats during habituation (Hab), after I6 days of cocaine escalation (Esc), and after abstinence (Abs). ${ }^{*} * * *<0.001$, significant difference relative to Hab. (c) Mean ( \pm SEM) number of cocaine injections per session during escalation and re-escalation of cocaine intake after abstinence neurons in Low (gray circles) and High CS (white circles) is plotted as a function of days. ${ }^{+} p<0.05$, significant difference relative to Low CS rats. 
a

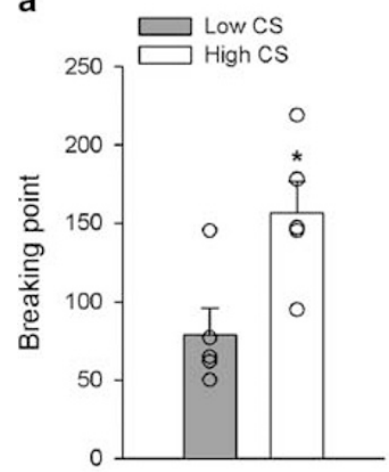

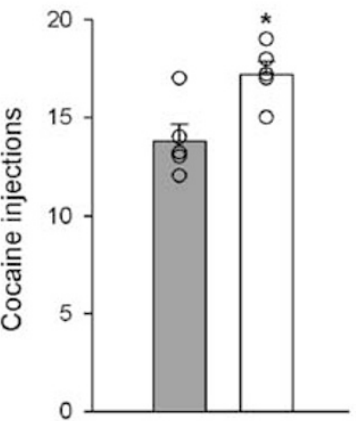

b

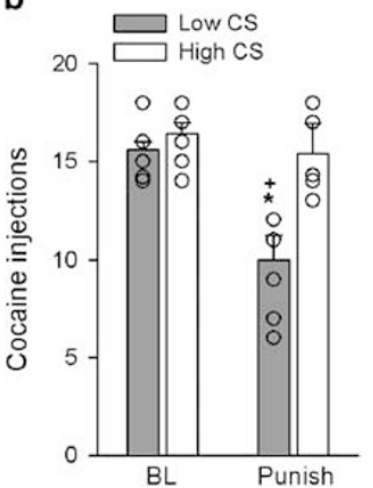

Figure 3 Increased cocaine-seeking behaviors in High CS rats. (a) Mean ( \pm SEM) number of the final ratio attained (breakpoint) and mean ( \pm SEM) number of cocaine injections under a PR schedule of reinforcement after abstinence in Low (gray bars) and High CS (white bars) rats. (b) Mean ( \pm SEM) of cocaine injections during a basal non-punished (BL) and punished sessions (Punish) in Low (gray bars) and High CS (white bars) rats. Each dot represents individual data point. $* p<0.05$, significant difference relative to Low CS animals. $+p<0.05$, significant difference relative to BL non-punished session.

cocaine cue was presented alone. This design allowed us to measure basal NAc neuronal reactivity to cues before the onset of cocaine intake. The total data set consisted of 256 NAc neurons (Hab: 96 neurons, Esc: 81 neurons, Abs: 79 neurons). Of these neurons, $25 \%$ (64/256) responded to the CS presentation during the cue-probe phase. Most of these CS responsive neurons $(78 \%, 50 / 64)$ phasically increased their firing rate, while only a small fraction $(22 \% ; 14 / 64)$ phasically decreased their firing. Importantly, the percentage of excitatory CS neurons increased between the first two phases and the last day of abstinence $(9 / 96,8 / 81$, and 19/79; Chi-square test: $\left.\chi^{2}=6.81 ; p<0.05\right)$, while the percentage of inhibitory CS neurons remained unchanged $(2 / 96,6 / 81$, and $6 / 79 ; \chi^{2}=3.1$; NS). Therefore, all subsequent analyses were conducted on excitatory CS responsive neurons.

We first determined incubation-like increase in NAc neuronal reactivity after abstinence by calculating the percentage of NAc phasic neuronal responses to cues during the cue-probe phase performed on the last day of abstinence. Rats were ranked according to their individual percentage of CS responsive neurons recorded during the last day of abstinence and split by the median into Low and High CS groups ( $n=5$ /group) (Figure 2a). Although the percentage of CS responsive neurons did not change during cocaine intake escalation, it considerably increased after abstinence but only in High CS rats (phase $\times$ group: $\mathrm{F}(2,16)=3.4 ; p<0.05$; Tukey $p<0.001$ ) (Figure 2b). We next assessed whether the higher level of CS responsive neurons seen in High CS rats could be predictive of an increased propensity for cocaine intake escalation (Figure 2c). ANOVA analysis revealed that cocaine intake escalation was different between the two groups of rats (day $\times$ group: $\mathrm{F}(23,184)=2.0 ; p<0.01)$. Further analysis indicated that cocaine intake gradually increased during cocaine self-administration sessions during the escalation (day effect: $\mathrm{F}(15,120)=32.4 ; p<0.001)$ and the re-escalation phases (day effect: $\mathrm{F}(4,56)=16.8 ; p<0.001$ ) in both Low and High CS rats. However, though not different during the escalation phase (group effect: $\mathrm{F}(1,8)=2.4$; NS), High CS rats self-administered more cocaine than Low CS rats during the re-escalation phase (group effect: $\mathrm{F}(1,8)=5.3$; $p<0.05$ ), indicating that a higher incubated NAc reactivity to cocaine cues predicts an increased re-escalation of cocaine self-administration after abstinence. Importantly, on the last day of escalation that preceded abstinence, both groups selfadministered the same total number of cocaine injections ( $F$ $(1,8)=0.6$; NS) and initiated the first lever pressing with the same latency $(F(1,8)=1.9$; NS), suggesting that postabstinence differences between High and Low CS rats did not preexist but rather built up during prolonged abstinence.

Because cocaine addiction is also characterized by a persistent increase in the motivation to seek and take the drug even after prolonged abstinence, we next assessed whether the incubation-like increase in NAc neuronal reactivity to the cocaine cues in High CS rats was associated with changes in the motivation for cocaine after abstinence. We used a progressive ratio schedule in which the number of responses (ratio) to obtain one drug injection progressively increases within the session, and we measured the breakpoint, the last ratio completed, which is considered a reliable index of the motivation for the drug (Figure 3a). We found that High CS rats worked harder than Low CS rats to obtain the drug as revealed by a higher breaking point for cocaine ( $\mathrm{F}$ $(1,8)=8.4 ; p<0.05)$ and obtained more cocaine injections $(\mathrm{F}$ $(1,8)=9.0 ; p<0.05)$. Finally, we assessed whether the incubation-like increase in NAc neuronal reactivity to the cocaine cues in High CS rats was associated with altered resistance to punishment. We measured the persistence of the animal in taking cocaine when it was signaled that its delivery would be associated with risk of punishment of $50 \%$ (Figure $3 \mathrm{~b}$ ). We found that High CS rats exhibited a higher resistance to risk of punishment $(\mathrm{F}(1,8)=5.1 ; p<0.05)$. Although the number of cocaine injections dropped during the punished session compared with baseline non-punished session in Low CS rats $(p<0.05)$, it remained stable in High CS rats, indicating that a higher NAc neuronal reactivity to cocaine cues predicts an increased propensity to continue to seek and/or obtain the drug after abstinence. Further investigations of the relationship between the incubationlike increase in NAc neuronal reactivity and drug-related behavior after abstinence revealed that the percentage of CS responsive neurons after abstinence was positively correlated with an increased re-escalation of cocaine intake $(r=0.66$; $p<0.05$; Figure 4a), an increased motivation for the drug $(r=0.76 ; p<0.05$; Figure $4 \mathrm{~b})$, and an increased resistance to 
a

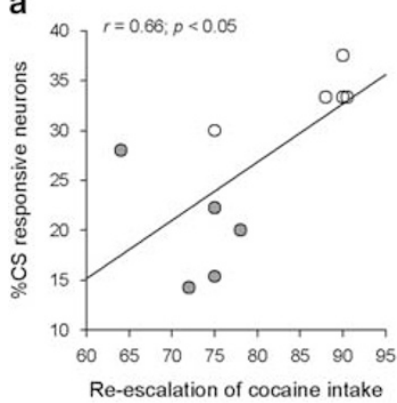

b

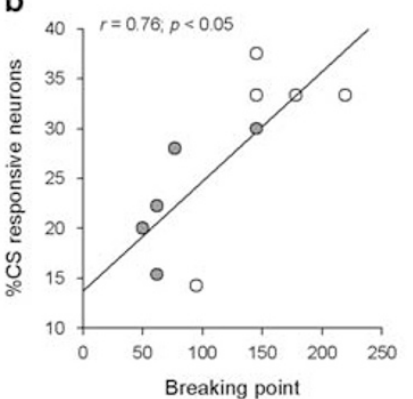

C

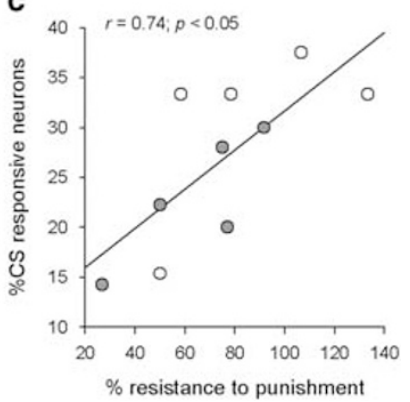

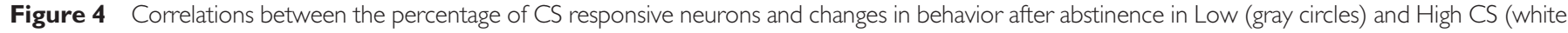

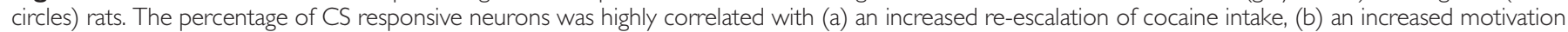
of the drug, and (c) an increased resistance to punishment after abstinence.

punishment after abstinence ( $r=0.74 ; p<0.05$; Figure $4 c)$. Importantly, the percentage of CS responsive neurons at the end of the escalation phase did not predict subsequent changes in drug-related behavior after abstinence, suggesting that the predictive effects of the incubation-like increase in NAc neuronal reactivity were selective. Specifically, individuals with CS responsive neurons above the median during the last session of escalation did not show increased reescalation of cocaine intake $(\mathrm{F}(1,8)=1.8$; NS), increased drug motivation $(\mathrm{F}(1,8)=0.11$; NS), or increased resistance to punishment $(\mathrm{F}(1,8)=0.14$; NS) compared with individuals with a ESC neuronal score below the median (data not shown).

\section{DISCUSSION}

Drug-associated stimuli can themselves act as powerful incentives promoting renewed drug seeking and taking after extinction or prolonged abstinence (de Wit and Stewart, 1981; Shaham et al, 2003). Therefore, we characterized NAc neuronal activity in response to non-contingent drugassociated cue presentation before and after 1 month of abstinence. Consistent with previous findings (Conrad et al, 2008; Hollander and Carelli, 2005), we found that incubation of cocaine seeking is associated with an increase in NAc neurons that encode drug-associated stimuli. However, there was some individual variability in the heightened NAc responses to cocaine cues. Specifically, a subgroup of rats (ie, High CS rats) showed a robust increase in NAc reactivity after abstinence, whereas another group did not (ie, Low CS rats). Most notably, increased NAc reactivity to drugassociated cues predicted an increased propensity to relapse into cocaine intake escalation after abstinence. That is, High CS rats with a higher postabstinence proportion of CS responsive neurons re-escalated their cocaine intake to a greater extent than Low CS rats. After re-escalation, High CS rats also worked harder and were less sensitive to risk of punishment than Low CS rats, indicating a strengthened motivation to seek and/or take the drug in that group of rats. Moreover, this incubation-like increase in NAc neuronal reactivity was positively correlated with both an increased reescalation of cocaine intake and an increased motivation for the drug after abstinence.

Importantly, during escalation of cocaine intake that preceded abstinence, each subgroup of rats self- administered the same total number of cocaine and showed the same percentage of CS responsive neurons. Thus the difference in incubation-like increase in NAc neuronal reactivity observed between the two groups cannot be explained by a preexisting difference in drug consumption or in initial neuronal reactivity to drug-associated cues. Rather, the increase in NAc neuronal reactivity in High CS rats appears to build-up during prolonged abstinence. This interpretation is further supported by the fact that the percentage of CS responsive neurons at the end of the escalation phase did not predict subsequent changes in drugrelated behavior after abstinence. At this stage, lack of evidence of a similar incubation-like process in neuronal reactivity in Low CS rats may be interpreted variously. It may indicate that Low CS rats are resilient to incubation-related neuronal processes during abstinence. However, one cannot rule out that such process did occur but eventually quickly recovered to normal within 1 month of abstinence. Alternatively, it is also possible that such process is slower to develop in Low CS rats and thus requires a longer period of abstinence to emerge. Future research with repeated measurement of NAc neuronal reactivity to drug CS during abstinence will be required to disentangle these different possibilities.

Though the cellular and synaptic mechanisms underlying heightened NAc cue reactivity after abstinence from cocaine have been thoroughly studied (Wolf, 2016), little is known about their functional significance. We have previously hypothesized that rats left undisturbed in their home cage during prolonged abstinence may rehearse their past experience with cocaine, thereby consolidating cocaine seeking-related memories (Guillem et al, 2014). This hypothesis is generally consistent with the fact that relapse can be attenuated by behavioral enrichment of the home cage during abstinence in rats and mice (Chauvet et al, 2009; Thiel et al, 2012). Accordingly, engagement in alternative activities during abstinence would divert, at least partly, animals from rehearsing their past experience with cocaine. Future research is needed to determine whether and to what extent behavioral enrichment of the home environment can impact the incubation-like increase in NAc neuronal reactivity seen here in High CS rats.

The identification of predictors of relapse is of high interest in human addiction research (Franken and Hendriks, 1999; Hattenschwiler et al, 2000; Miller, 1996). 
Currently, addiction research is focusing more on the role of neurocognitive predictors (Garavan and Hester, 2007; Goldstein and Volkow, 2011). For instance, attentional bias, which refers to the automatically enhanced cognitive processing of drug-related (salient) stimuli compared with neutral (non-salient) ones has recently been associated with craving (Field and Cox, 2008; Franken, 2003) and shown to predict vulnerability to relapse into cocaine addiction (Carpenter et al, 2006; Marhe et al, 2013). Brain regions that have been found to be associated with attentional bias in addiction include the anterior cingulate cortex (Luijten et al, 2012; Luijten et al, 2011), the dorsolateral prefrontal cortex (Hester and Garavan, 2009; Luijten et al, 2012; VollstadtKlein et al, 2012), and subcortical regions, such as the NAc (Nestor et al, 2011). Consistent with this, we found that the incubation of NAc reactivity to drug cues predicted subsequent vulnerability to relapse into cocaine intake escalation.

In conclusion, this study shows that postabstinence increase in NAc cue reactivity represents a neurobiological marker that predicts the vulnerability to relapse after prolonged abstinence and suggests that assessment of NAc cue reactivity during abstinence may help to identify individuals at risk for relapse. The development of strategies to reverse, compensate, or prevent abstinence-induced increase in NAc neuronal reactivity may eventually prove useful to further improve relapse prevention.

\section{FUNDING AND DISCLOSURE}

This work was supported by the French Research Council (CNRS), the Université de Bordeaux, and the French National Agency (ANR-2010-BLAN-1404-01). The authors declare no conflict of interest.

\section{REFERENCES}

Carpenter KM, Schreiber E, Church S, McDowell D (2006). Drug Stroop performance: relationships with primary substance of use and treatment outcome in a drug-dependent outpatient sample. Addict Behav 31: 174-181.

Chauvet C, Lardeux V, Goldberg SR, Jaber M, Solinas M (2009). Environmental enrichment reduces cocaine seeking and reinstatement induced by cues and stress but not by cocaine. Neuropsychopharmacology 34: 2767-2778.

Conrad KL, Tseng KY, Uejima JL, Reimers JM, Heng LJ, Shaham Y et al (2008). Formation of accumbens GluR2-lacking AMPA receptors mediates incubation of cocaine craving. Nature 454: $118-121$.

Dackis CA, O'Brien CP (2001). Cocaine dependence: a disease of the brain's reward centers. J Subst Abuse Treat 21: 111-117.

de Wit H, Stewart J (1981). Reinstatement of cocaine-reinforced responding in the rat. Psychopharmacology (Berl) 75: 134-143.

Ferrario CR, Loweth JA, Milovanovic M, Ford KA, Galinanes GL, Heng LJ et al (2011). Alterations in AMPA receptor subunits and TARPs in the rat nucleus accumbens related to the formation of $\mathrm{Ca}(2)(+)$-permeable AMPA receptors during the incubation of cocaine craving. Neuropharmacology 61: 1141-1151.

Field M, Cox WM (2008). Attentional bias in addictive behaviors: a review of its development, causes, and consequences. Drug Alcohol Depend 97: 1-20.

Franken IH (2003). Drug craving and addiction: integrating psychological and neuropsychopharmacological approaches. Prog Neuropsychopharmacol Biol Psychiatry 27: 563-579.
Franken IH, Hendriks VM (1999). Predicting outcome of inpatient detoxification of substance abusers. Psychiatr Serv 50: 813-817.

Garavan H, Hester R (2007). The role of cognitive control in cocaine dependence. Neuropsychol Rev 17: 337-345.

Gawin FH (1991). Cocaine addiction: psychology and neurophysiology. Science 251: 1580-1586.

Goldstein RZ, Volkow ND (2011). Dysfunction of the prefrontal cortex in addiction: neuroimaging findings and clinical implications. Nat Rev Neurosci 12: 652-669.

Grimm JW, Hope BT, Wise RA, Shaham Y (2001). Neuroadaptation. Incubation of cocaine craving after withdrawal. Nature 412: 141-142.

Guillem K, Ahmed SH, Peoples LL (2014). Escalation of cocaine intake and incubation of cocaine seeking are correlated with dissociable neuronal processes in different accumbens subregions. Biol Psychiatry 76: 31-39.

Hattenschwiler J, Ruesch P, Hell D (2000). Effectiveness of inpatient drug detoxification: links between process and outcome variables. Eur Addict Res 6: 123-131.

Hester R, Garavan H (2009). Neural mechanisms underlying drugrelated cue distraction in active cocaine users. Pharmacol Biochem Behav 93: 270-277.

Hollander JA, Carelli RM (2005). Abstinence from cocaine selfadministration heightens neural encoding of goal-directed behaviors in the accumbens. Neuropsychopharmacology 30: 1464-1474.

Hollander JA, Carelli RM (2007). Cocaine-associated stimuli increase cocaine seeking and activate accumbens core neurons after abstinence. J Neurosci 27: 3535-3539.

Kearns DN, Weiss SJ, Panlilio LV (2002). Conditioned suppression of behavior maintained by cocaine self-administration. Drug Alcohol Depend 65: 253-261.

Lu L, Grimm JW, Hope BT, Shaham Y (2004). Incubation of cocaine craving after withdrawal: a review of preclinical data. Neuropharmacology 47(Suppl 1): 214-226.

Luijten M, Veltman DJ, Hester R, Smits M, Pepplinkhuizen L, Franken IH (2012). Brain activation associated with attentional bias in smokers is modulated by a dopamine antagonist. Neuropsychopharmacology 37: 2772-2779.

Luijten M, Veltman DJ, van den Brink W, Hester R, Field M, Smits $M$ et al (2011). Neurobiological substrate of smoking-related attentional bias. Neuroimage 54: 2374-2381.

Marhe R, Waters AJ, van de Wetering BJ, Franken IH (2013). Implicit and explicit drug-related cognitions during detoxification treatment are associated with drug relapse: an ecological momentary assessment study. J Consult Clin Psychol 81: $1-12$.

McCutcheon JE, Wang X, Tseng KY, Wolf ME, Marinelli M (2011). Calcium-permeable AMPA receptors are present in nucleus accumbens synapses after prolonged withdrawal from cocaine self-administration but not experimenter-administered cocaine. J Neurosci 31: 5737-5743.

Miller WR (1996). What is a relapse? Fifty ways to leave the wagon. Addiction 91(Suppl): S15-S27.

Nestor L, McCabe E, Jones J, Clancy L, Garavan H (2011). Differences in "bottom-up" and "top-down" neural activity in current and former cigarette smokers: Evidence for neural substrates which may promote nicotine abstinence through increased cognitive control. Neuroimage 56: 2258-2275.

Richardson NR, Roberts DC (1996). Progressive ratio schedules in drug self-administration studies in rats: a method to evaluate reinforcing efficacy. J Neurosci Methods 66: 1-11.

See RE (2002). Neural substrates of conditioned-cued relapse to drug-seeking behavior. Pharmacol Biochem Behav 71: 517-529.

Shaham Y, Shalev U, Lu L, De Wit H, Stewart J (2003). The reinstatement model of drug relapse: history, methodology and major findings. Psychopharmacology (Berl) 168: 3-20. 
Shalev U, Grimm JW, Shaham Y (2002). Neurobiology of relapse to heroin and cocaine seeking: a review. Pharmacol Rev 54: 1-42.

Sinha R, Fuse T, Aubin LR, O'Malley SS (2000). Psychological stress, drug-related cues and cocaine craving. Psychopharmacology (Berl) 152: $140-148$

Thiel KJ, Painter MR, Pentkowski NS, Mitroi D, Crawford CA, Neisewander JL (2012). Environmental enrichment counters cocaine abstinence-induced stress and brain reactivity to cocaine cues but fails to prevent the incubation effect. Addict Biol 17: 365-377.
Vanderschuren LJ, Everitt BJ (2004). Drug seeking becomes compulsive after prolonged cocaine self-administration. Science 305: 1017-1019.

Vollstadt-Klein S, Loeber S, Richter A, Kirsch M, Bach P, von der Goltz C et al (2012). Validating incentive salience with functional magnetic resonance imaging: association between mesolimbic cue reactivity and attentional bias in alcohol-dependent patients. Addict Biol 17: 807-816.

Wolf ME (2016). Synaptic mechanisms underlying persistent cocaine craving. Nat Rev Neurosci 17: 351-365. 